\title{
The Preparation of Large-scale Synchronous Cultures of the Trypanosomatid, Crithidia fasciculata, by Cell-size Selection: Changes in Respiration and Adenylate Charge through the Cell-cycle
}

\author{
By C. EDWARDS,* M. STATHAM AND D. LLOYD \\ Department of Microbiology, University College, \\ Cardiff $\mathrm{CF}_{2}$ ITA
}

(Received 30 October 1974; revised 28 December 1974)

\begin{abstract}
SUMMARY
Large-scale synchronous cultures of Crithidia fasciculata were prepared by a sedimentation-velocity-size-selection method using a non-osmotic gradient in a zonal rotor. Synchrony indices of up to 0.68 were obtained (average length of cell-cycle, $5 \cdot \mathrm{I}$ h). Dry weight, protein and RNA increased continuously so as to double in one cell-cycle. Rates of oxygen uptake $/ \mathrm{ml}$ culture increased overall so as to double over a cell-cycle, but rose to maxima at five periods in the cellcycle. KCN gave a similar degree of inhibition throughout, and so did not alter the periodicity or amplitude of the oscillations. Total adenylates doubled over a cycle, and complex changes in pool sizes of ATP, ADP and AMP were temporally interrelated and were correlated with changes of oxygen uptake rates rather than with changes in biosynthetic requirements. Adenylate charge varied between 0.47 and 0.66 . Discontinuous respiratory activity of mitochondria through the cellcycle and possible mechanisms for its control are discussed with reference to previous data.
\end{abstract}

\section{INTRODUCTION}

Investigations on asynchronous cultures of trypanosomes, which are organisms having a single large mitochondrion (Simpson, 1972), have revealed a close temporal connection of nuclear and mitochondrial (kinetoplast) DNA synthesis, and of nuclear and kinetoplast division (Steinert \& Steinert, 1962; Steinert \& Van Assel, 1967; Cosgrove \& Skeen, 1970). The existence of a discrete S-phase for kinetoplast DNA and its synchronicity with the S-phase of nuclear DNA in these organisms makes them unique systems for the study of the control of mitochondrial biogenesis; a clear relationship between these events has not been reported in other eukaryotic micro-organisms (Lloyd, 1974). Further experiment on the trypanosome system requires a method for the preparation of synchronous cultures which gives minimal metabolic disturbance. Previous reports of partially synchronized cultures of various species of trypanosomes are not free from criticism, in that they all involve induction methods. Furthermore, the degree of synchrony achieved was not completely satisfactory. Hydroxyurea treatment has been used to induce partial synchrony of Crithidia luciliae (Steinert, 1969; Van Assel \& Steinert, 1971) and of Leishmania tarentolae (Simpson \& Braly, 1970); temperature shocks induced partial synchrony in C. oncopelti (Newton, 1957).

We report the development of a method for producing large-scale synchronous cultures

* Present address: Department of Biochemistry, University of Leicester, Leicester LEI 7 RH. 
of $C$. fasciculata, based on the velocity-sedimentation procedure of Mitchison \& Vincent (I965), which does not suffer from the disadvantages of induced synchrony methods (Mitchison, I97I). The resulting cultures exhibit 'balanced growth' (Campbell, 1957), in that dry weight, total protein and RNA (all expressed as per millilitre of culture) increase continuously so as to double during one cell-cycle. Respiration rates and intracellular pools of adenine nucleotides show complex oscillatory changes.

\section{METHODS}

Growth and harvesting of the organism. Cultures of Crithidia fasciculata were maintained, grown, counted and harvested as previously described (Edwards \& Lloyd, I973), except that harvesting was at room temperature. Glycerol was the major energy source.

Preparation of synchronous cultures. The velocity-sedimentation procedure for the sizeselection of organisms finally adopted was based on that of Mitchison \& Vincent (1965), but utilized the greater capacity offered by a zonal rotor (Poole, Lloyd \& Kemp, I973), and was further modified by employing a non-osmotic gradient material (Dextran dissolved in growth medium). A linear gradient ( 3 to $12 \%, \mathrm{w} / \mathrm{v}$; total volume $540 \mathrm{ml}$ ) was generated using an Isco Dialagrad pump (Shandon Southern Instruments Ltd, Camberley, Surrey) and loaded into an HS zonal rotor running in a High Speed 18 centrifuge (MSE Ltd, Crawley, Sussex) at $600 \mathrm{rev} . / \mathrm{min}$. Accurate control of rotor speed was by means of a 'low-speed zonal control' ancillary circuit box. The remainder of the rotor volume was filled with $60 \%(\mathrm{w} / \mathrm{w})$ sucrose. The culture $(61)$ was harvested at room temperature, resuspended in $70 \mathrm{ml}$ distilled water, and homogenized (3 strokes) in a loose-fitting hand homogenizer (Jencons Scientific Ltd, Hemel Hempstead, Hertfordshire). The cell suspension was pumped on to the gradient $(40 \mathrm{ml} / \mathrm{min})$, followed by an overlay of $30 \mathrm{ml}$ distilled water. The rotor was accelerated to $1450 \mathrm{rev} . / \mathrm{min}$ and held at this speed until the band of cells had become distributed across the gradient (about $6 \mathrm{~min}$ ). Unloading was achieved by displacement with $60 \%$ sucrose $(30 \mathrm{ml} / \mathrm{min})$ after deceleration to $600 \mathrm{rev} . / \mathrm{min}$; Io $\mathrm{ml}$ fractions were collected. The value of the integrated time-field for this procedure was:

$$
\int_{0}^{t} \omega^{2} \mathrm{~d} t=3 \cdot 6 \times \mathrm{Io}^{9} \mathrm{rad}^{2} / \mathrm{s}
$$

Fractions ( 30 to $40 \mathrm{ml}$ total volume) containing rapidly sedimenting organisms were used to inoculate $200 \mathrm{ml}$ to $\mathrm{I} l$ of growth medium containing carbenicillin $(0.5 \mathrm{mg} / \mathrm{ml})$ so as to give an initial population of $5 \times 10^{6}$ to $1 \cdot 5 \times 10^{7}$ organisms $/ \mathrm{ml}$. Control experiments indicated that this antibiotic is without effect on the growth of the organism.

Assessment of synchrony. Numbers of organisms were measured periodically and the degree of synchrony assessed by the synchrony index of Blumenthal \& Zahler (1962), calculated from the equation:

$$
F=\left(N / N_{0}\right)-2^{t / g},
$$

where $F$ has a maximum value of $\mathrm{I} \cdot \mathrm{O}$ in a culture exhibiting theoretically perfect synchrony, $N$ is the number of organisms at time $t, N_{0}$ the number of organisms at zero time, and $g$ the mean generation time.

In the presentation of results, vertical lines indicate the mid-points of doublings in cell numbers.

Analytical methods. Dry weight was determined on organisms harvested from $50 \mathrm{ml}$ samples of culture after washing in $20 \mathrm{~mm}$-potassium phosphate buffer $\mathrm{pH} 7 \cdot 4$. Total cell 
protein was measured on organisms from $8 \mathrm{ml}$ samples of culture by the method of Herbert, Phipps \& Strange (I97I), using bovine serum albumin as a standard. Total RNA was determined on organisms from $50 \mathrm{ml}$ samples of culture by the orcinol method of Herbert et al. (I97I), using yeast RNA as a standard. Polarographic measurements of oxygen uptake by undiluted whole-cell suspensions were made with an oxygen electrode (Lloyd \& Brookman, I967); errors on replicate determinations were less than $4 \%$. Measurements of adenine nucleotide levels were made using luciferase assays in a specially constructed photometer utilizing a low-background photomultiplier (EMI $9524 \mathrm{~S}$ ) attached to a Vitatron recorder (Dieren, The Netherlands). Extracts were prepared from I $\mathrm{ml}$ culture samples by either perchlorate (Chapman, Fall \& Atkinson, 1971) or butanol extraction (Chiu et al. 1973). Estimations of ATP, ADP and AMP were by the methods of Chapman et al. (I97I) using ATP as a calibration standard. The lower limit of sensitivity of the ATP assay was $0.1 \mathrm{pmol}$ in a total volume of $0.2 \mathrm{I} \mathrm{ml}$; errors on replicate determinations were less than I \%. 'Adenylate charge' (Atkinson \& Walton, 1967) is defined as:

$$
\text { Adenylate charge }=\frac{\mathrm{ATP}+\frac{1}{2} \mathrm{ADP}}{\mathrm{ATP}+\mathrm{ADP}+\mathrm{AMP}} .
$$

Chemicals. Dextran (Chemical grade, average mol. wt 82000), RNA (grade VI, ex Torula yeast), buffered firefly lantern extract $(50 \mathrm{mg}$ dried lanterns/vial), myokinase (grade III, rabbit muscle), pyruvate kinase (type II, rabbit skeletal muscle), phosphoenolpyruvic acid (sodium salt) and ATP (disodium salt) were all obtained from Sigma. Sucrose was mineral-water sugar from Tate and Lyle Ltd, Cardiff. Bovine serum albumin was from Armour Pharmaceutical Co., Eastbourne, Sussex, and carbenicillin from Beecham Research Laboratories, Brentford, Middlesex.

\section{RESULTS}

\section{Development of methods for establishing synchronous cultures}

Attempts to select organisms from an exponentially growing population on the basis of size were made using linear sucrose gradients (IO to $60 \%, w / w$, made up in growth medium). Fig. I $(a)$ shows the bimodal distribution of cells produced by rate-zonal separation. Inoculation of organisms from zones $\mathbf{B}$ and $\mathrm{C}$ into fresh growth medium was followed by synchronous bursts of cell division after $3 \mathrm{~h}$ (Fig. I $b$ ) and $2 \mathrm{~h}$ (Fig. I $c$ ), respectively. The most slowly sedimenting cells from zone B showed only a $28 \%$ increase in numbers over a $\mathrm{I} h$ division period; these figures give a synchrony index of only $\mathrm{O} \cdot \mathrm{I} 2$. The more-rapidly sedimenting cells from zone $\mathrm{C}$ gave a perfect doubling in numbers over a $2.5 \mathrm{~h}$ division period, corresponding to a synchrony index of 0.58 for the first division; the second division was also synchronous. Examination by light microscopy revealed the presence of clusters ('rosettes'; Brooker, 197I) of organisms in all fractions. Large rosettes composed of many hundreds of organisms were seen in the major rapidly sedimenting zone.

In order to minimize rosette formation, which is thought to be an osmotically dependent phenomenon (Brooker, I97I), the cells were resuspended in distilled water and sedimented through a Dextran gradient as described in Methods. Figure 2 shows the distribution of organisms in a typical experiment, and the region of the gradient used for inoculation of cultures in all subsequent work. 

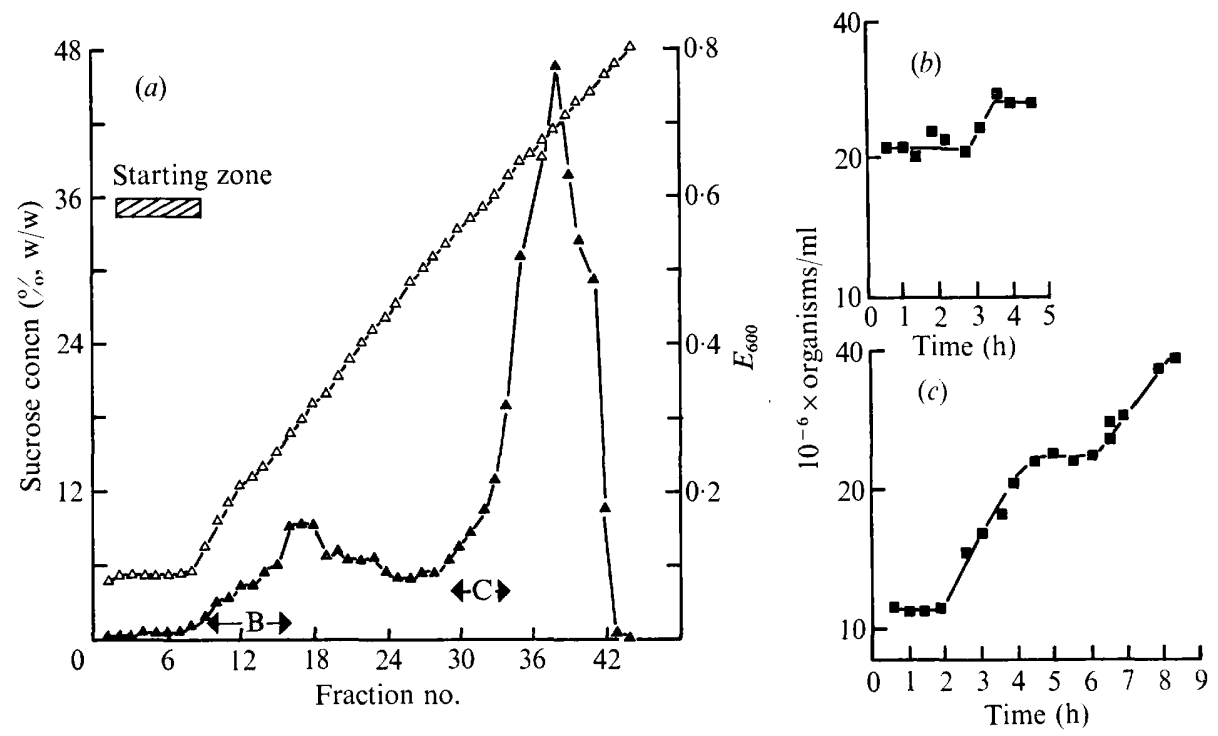

Fig. I. Size selection of cells from an exponentially growing culture of $C$. fasciculata by rate-zonal centrifugation through a sucrose gradient made up in growth medium, and the subsequent growth kinetics of selected fractions. A total of $3.3 \times 10^{11}$ organisms suspended in growth medium was loaded on to a linear sucrose gradient (also in growth medium) in an MSE HS zonal rotor. Centrifugation was at $1450 \mathrm{rev}$. $/ \mathrm{min}$ for $6 \mathrm{~min}\left(900 \mathrm{~g}\right.$ minimum at sample zone, $\left.\int_{0}^{t} \omega^{2} \mathrm{~d} t=3.57 \times \mathrm{IO}^{9} \mathrm{rad}^{2} / \mathrm{s}\right)$. Fractions ( $10 \mathrm{ml}$ ) were collected at room temperature. (a) $\Delta, E_{600} ; \Delta$, sucrose concentration. (b) Growth of organisms from fractions 9 to $16(\mathrm{~B})$, and (c) fractions 32 to 42 (C), after inoculation into sterile growth medium $(200 \mathrm{ml})$.

\section{Measurements of total protein and RNA through the cell-cycle}

After a lag of $\mathrm{I} \cdot 5 \mathrm{~h}$ cell numbers showed a $94 \%$ increase over a $\mathrm{I} \cdot 5 \mathrm{~h}$ division period in a cell-cycle of $5 . \mathrm{I} h$, giving a synchrony index of 0.66 (Fig. 3). Total protein and RNA/ml culture increased continuously so as to double during one cell-cycle (Fig. 3).

\section{Measurements of dry weight, oxygen uptake rates and the effect of cyanide}

In another experiment the synchrony index was 0.68 (Fig. 4, curve A). Dry weight $/ \mathrm{ml}$ culture (Fig. 4, curve B) increased so as to double over one cell-cycle. Rates of respiration of undiluted samples, removed at frequent intervals from the growth vessel, also doubled overall during one cell-cycle, but superimposed on this increase was a complex oscillatory component (Fig. 4, curve C). The period of this component was approximately one-fifth of a cell-cycle, and its amplitude corresponded to between 8 and $16 \%$ of the average cellular respiration. Addition of $\mathrm{KCN}$ at a concentration $(0.042 \mathrm{~mm})$ sufficient to give $30 \%$ inhibition of respiration of an exponential culture (Edwards \& Lloyd, 1973), similarly inhibited samples taken at either respiratory maxima or minima. The inhibited respiration (Fig. 4, curve D) therefore showed an oscillation similar in frequency, and of similar amplitude to that of the uninhibited respiration. Further addition of $\mathrm{KCN}$ (to $0.84 \mathrm{~mm}$ ) did not attenuate or damp the oscillating component (Fig. 4, curve E).

\section{Measurement of adenine nucleotide pools and calculation of adenylate charge}

Preliminary experiments indicated the butanol method of extraction of adenine nucleotides was more satisfactory than the perchloric acid method for C. fasciculata. Figure 5 


\section{Starting zone}

\section{बIVIV}

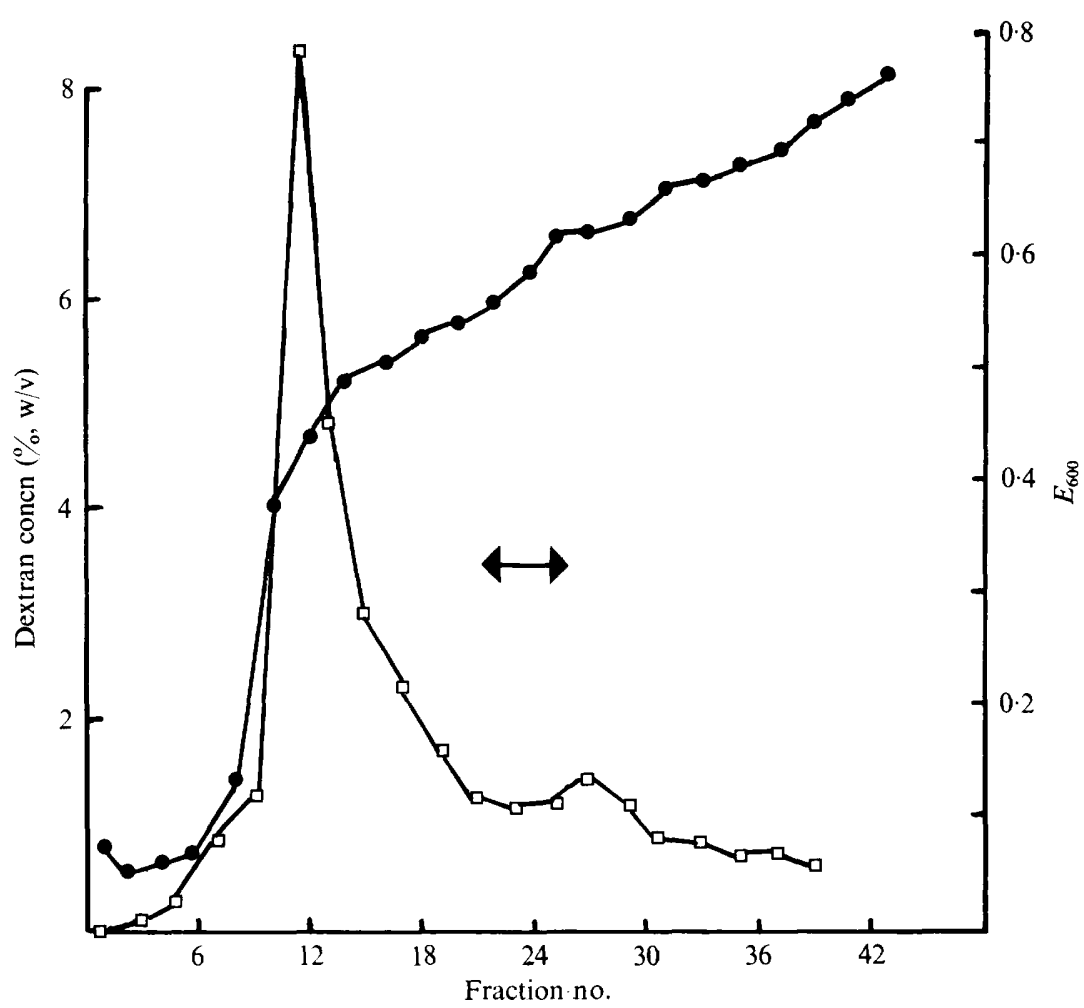

Fig. 2. Size selection of cells from an exponentially growing culture of $C$. fasciculata by rate-zonal centrifugation through a Dextran gradient made up in growth medium. A total of $3 \cdot 2 \times 10^{11}$ organisms suspended in distilled water was loaded on to a Dextran gradient in an MSE HS zonal rotor. Centrifugation was at $1450 \mathrm{rev} / \mathrm{min}$ for $6 \mathrm{~min}\left(900 \mathrm{~g}\right.$ minimum at sample zone, $\int_{0}^{t} \omega^{2} \mathrm{~d} t=3.57 \times 10^{9}$ $\operatorname{rad}^{2} / \mathrm{s}$ ). Fractions ( $10 \mathrm{ml}$ ) were collected at room temperature. $\square, E_{600} ; \boldsymbol{\ominus}$, Dextran concentration. Fractions 21 to 25 (indicated by double arrow) inoculated into fresh sterile growth medium gave the synchronous cultures routinely used in subsequent experiments.

shows the variations in ATP, ADP, total adenylates, and adenylate charge during a period equivalent to the generation time $(4 \cdot 75 \mathrm{~h})$ of an early exponential culture. ATP remained constant over this period (curve B), whereas ADP increased by $90 \%$ (curve C) and total adenylates by $62 \%$ (curve D). Values calculated for adenylate charge (curve E) remained constant at 0.66 for the first $2 \mathrm{~h}$, followed by a slight decrease to $0.6 \mathrm{r}$. The changes in pool size of AMP were too small to have a significant contribution to changes in the adenylate charge. Figure 6 shows the complex changes in adenine nucleotide pools in a culture which showed a synchrony index of 0.46 . As in the exponential culture (Fig. 5, curve B), the overall increase in the ATP pool (Fig. 6, curve B) did not follow the increase in cellular dry weight (Fig. 4, curve B), but showed periodic fluctuations. ADP/ml culture (Fig. 6, curve $\mathrm{C}$ ), however, doubled overall during a period of one cell-cycle and also showed complex periodic changes. AMP (Fig. 6, curve D) varied most as a percentage of the 


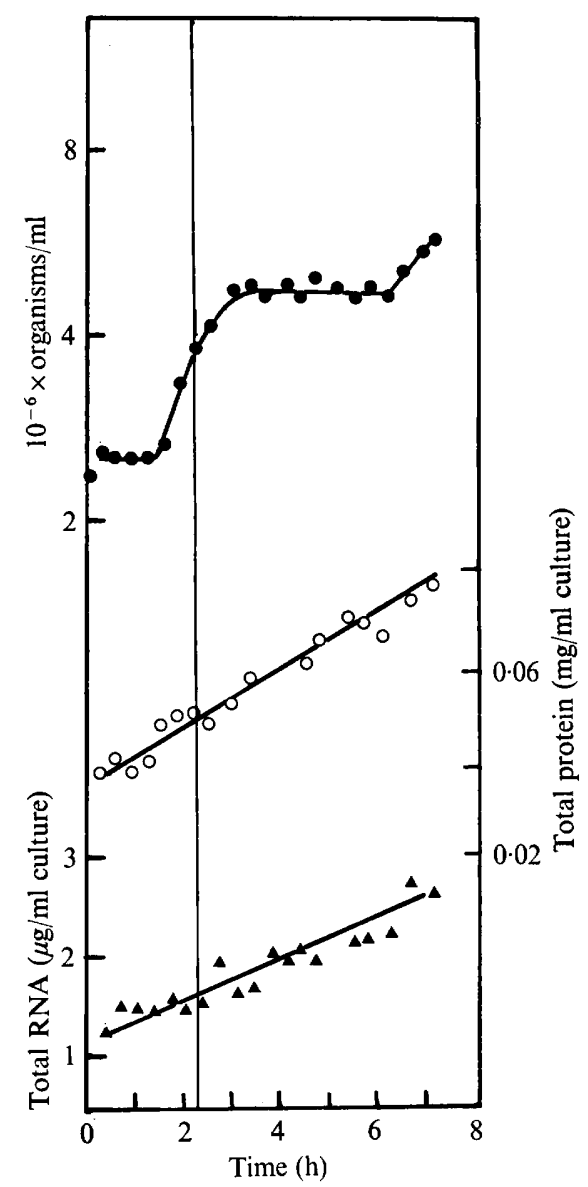

Fig. 3.

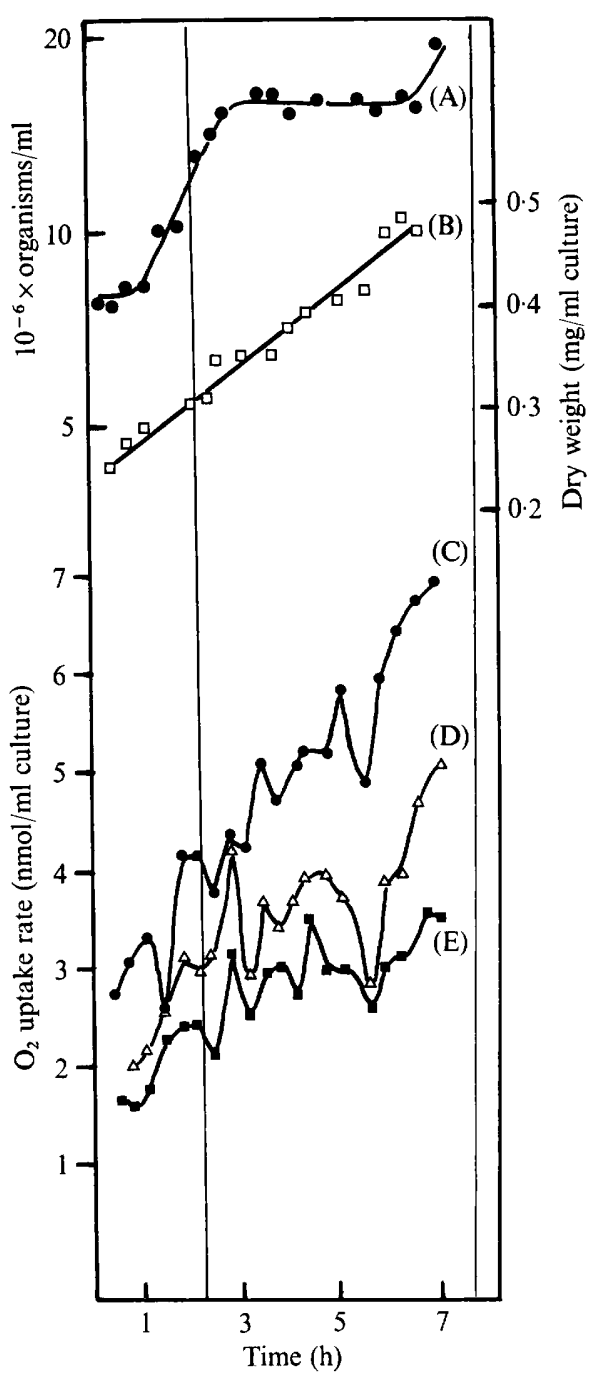

Fig. 4.

Fig. 3. Changes in total protein and total RNA in synchronous cultures of $C$. fasciculata. 9 , Cell numbers; $O$, total protein; $\boldsymbol{\Delta}$, total RNA.

Fig. 4. Oxygen uptake from a synchronous culture of $C$. fasciculata and the effect of $\mathrm{KCN}$. Growth of the culture was followed through a complete cell-cycle (A), and dry weight (B) increased continuously. Measurements of oxygen uptake rates were made on undiluted samples removed from the culture in the absence (C), or in the presence (D) of $0.042 \mathrm{mM}-\mathrm{KCN}$, or in the presence of $0.083 \mathrm{mM}-\mathrm{KCN}(\mathrm{E})$.

mean cellular content, going from a level below the lower limit of sensitivity of the assay to $0.75 \mathrm{nmol} / \mathrm{ml}$ culture. Total adenylates (Fig. 6, curve E) doubled overall during the cell-cycle and had an oscillating component. Over the first $\mathrm{I} \cdot 25 \mathrm{~h}$, adenylate charge (Fig. 6 , curve F) showed a dramatic fall from 0.75 . This was followed by an oscillation of characteristic pattern, with each of the three maxima being preceded by a distinct shoulder; during this period, adenylate charge varied between 0.66 and 0.47 . 


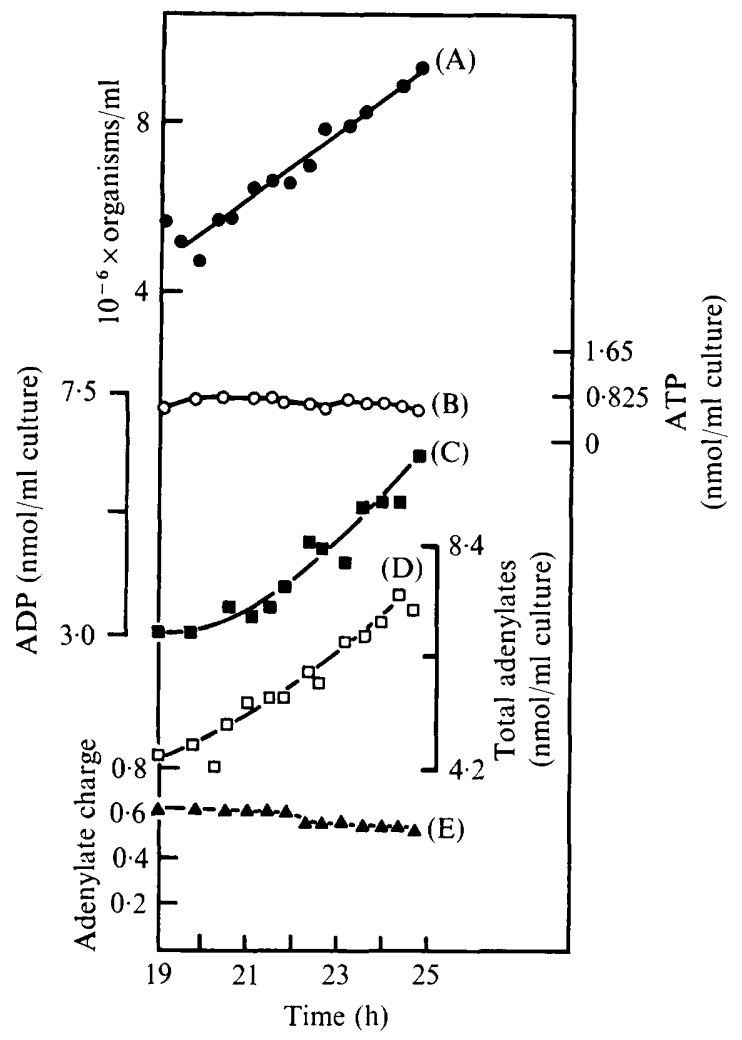

Fig. 5. Changes in adenine nucleotide pools, total adenylates and adenylate charge during the exponential growth of an asynchronous culture of $C$. fasciculata. Samples (I ml) were removed from an early exponential-phase batch culture and rapidly mixed with I $\mathrm{ml} n$-butanol. (A) Cell numbers, (B) ATP, (C) ADP, (D) total adenylates and (E) adenylate charge.

\section{DISCUSSION}

Three major difficulties have been encountered and overcome during attempts to prepare synchronous cultures of $C$. fasciculata by a size-selection procedure. The first is the sensitivity of the organism to media of high osmotic pressure; marked changes in size and shape occur during sedimentation through sucrose gradients. A second phenomenon which may have an osmotically controlled basis is the organized grouping of organisms nto rosettes. This occurs naturally in all cultures, and makes size-separation of individual organisms difficult. Rosette formation can be minimized by resuspending organisms in distilled water, but some loss of organisms into rosettes, which then rapidly sediment through the non-osmotic gradient, still occurs. The third difficulty is that the smallest cells of $C$. fasciculata (corresponding to $6 \%$ of the total population) appear to be nonviable, as shown by the imperfect synchrony obtained when they are used to start synchronous cultures. These abnormal cells are not produced as a result of the experimental procedures, since an independent method of size-selection which does not involve a density gradient step, and which does not damage other organisms known to be fragile, gave identical results (Lloyd et al. 1975). A possible explanation for the non-viability of the smallest cells is that these represent a naturally-occurring dyskinetoplastic population, similar 


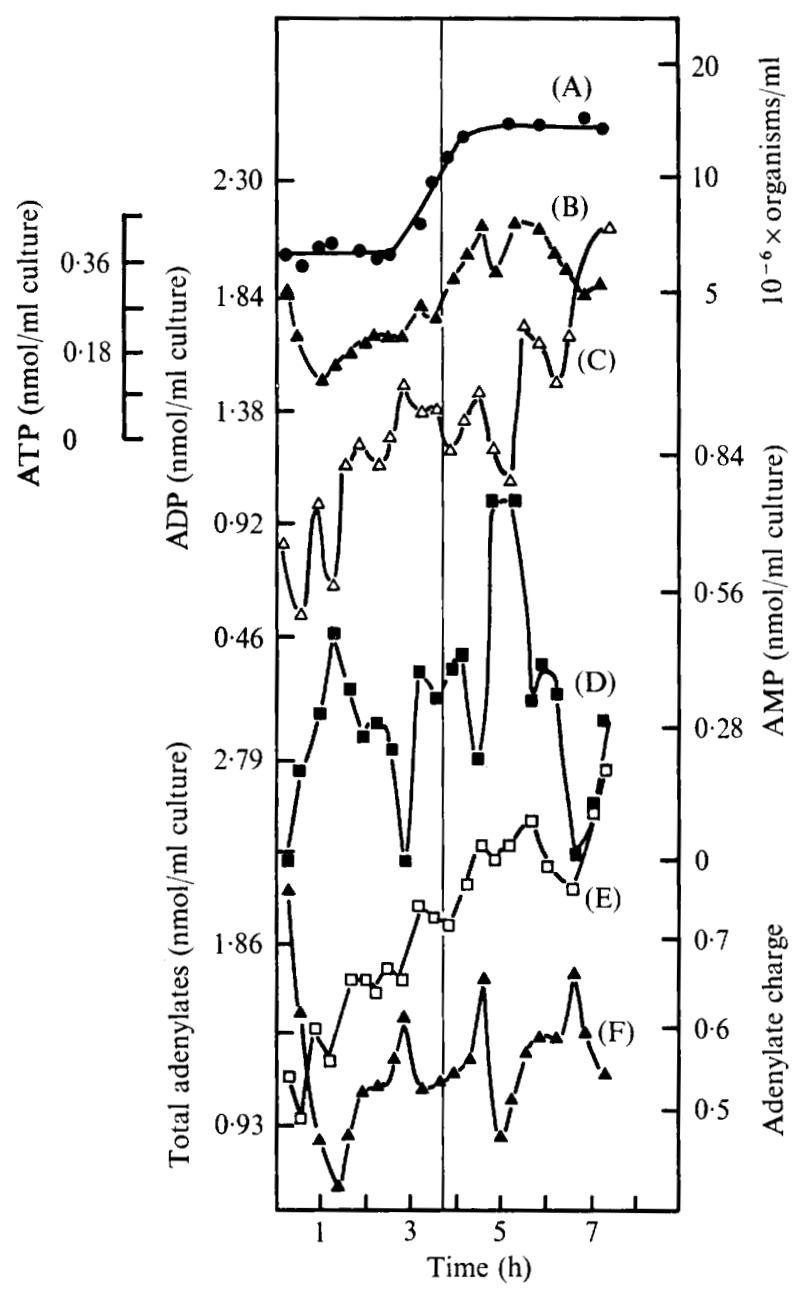

Fig. 6. Changes in adenine nucleotide pools, total adenylates and adenylate charge during synchronous growth of $C$. fasciculata. Samples ( $\mathrm{I} \mathrm{ml}$ ) were removed at frequent intervals from the culture and rapidly mixed with $I \mathrm{ml} n$-butanol. (A) Cell numbers, (B) ATP, (C) ADP, (D) AMP, (E) total adenylates and (F) adenylate charge.

to the $3 \%$ of the cells of an exponentially-growing culture of Leptomonas karyophilus which lack kinetoplasts (Stuart \& Hanson, 1967).

Of the four previous reports of the establishment of synchrony in trypanosomes, it is not possible to calculate synchrony indices from the data presented except in the case of C. oncopelti (Newton, I957), where heat-induced synchrony resulted in a $70 \%$ increase in cell numbers over a period equal to one-quarter of the mean generation time (synchrony index $=0.57$ ). The present method gives cultures with a high degree of synchrony in the absence of excessive metabolic disturbance, as shown by the observation that some of the criteria of 'balanced growth' are met during the first cell-cycle (for example, dry weight, protein and RNA in the culture double in amounts). The only indication of abnormal metabolism that we have detected is the aberrantly high value of adenylate 


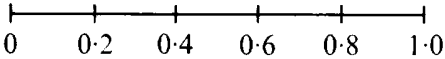

$Q_{\mathrm{O}_{2}}$

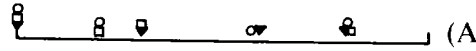

ATP

$8 \Delta \circ$ कि

ADP

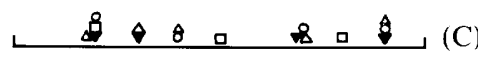

AMP

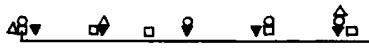

\section{Adenylate}

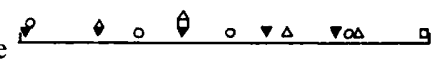

charge

Fig. 7. Cell-cycle maps of timings of maxima of oxygen uptake, ATP, ADP and AMP pools and adenylate charge in synchronous cultures of $C$. fasciculata. The abscissa represents the cell-cycle as a period $O$ to $\mathrm{I} \cdot \mathrm{O}$, the mid-point in doubling of cell numbers being taken as $\mathrm{I} \cdot \mathrm{O}$ when expressing events before cell division and as o when expressing events subsequent to cell division. (A) Symbols denote timings of maximal respiration rates as observed in four separate experiments (synchrony indices: $0.63,0.43,0.64$ and 0.63 ). Symbols denote timings of maximal pool sizes of ATP, ADP, AMP, and in adenylate charge, respectively: $\square, 0$, after perchloric acid extraction; $\triangle, \nabla$, after butanol extraction (synchrony indices: $0.62,0.47,0.63$ and 0.47 ).

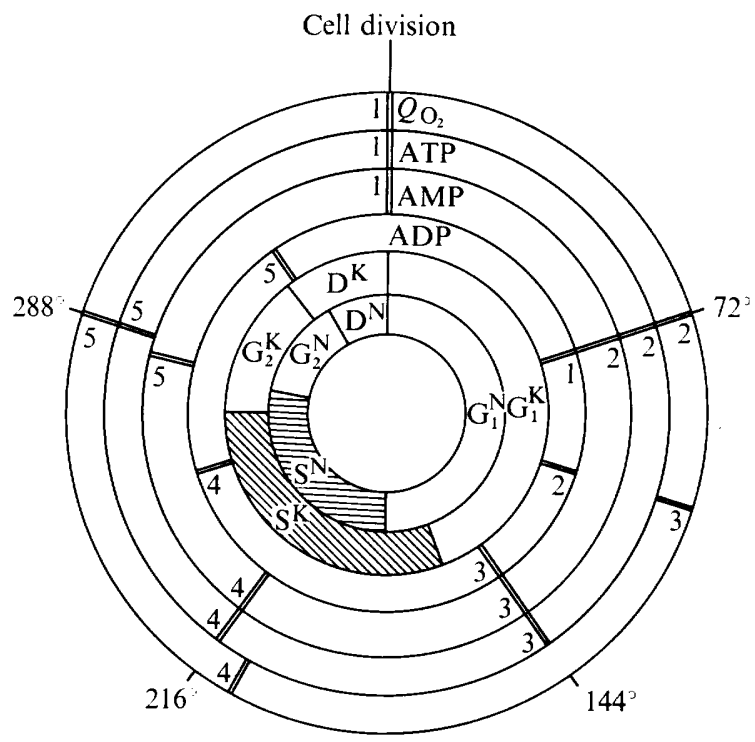

Fig. 8. Circular cell-cycle map for the timings of consistently observed maxima of oxygen uptake rates and adenine nucleotide pools in $C$. fasciculata. The phase angles of the oscillatory components are plotted with respect to cell division (at a phase angle of $0^{\circ}$ ). The phases of nuclear and kinetoplast events are replotted from the data of Cosgrove \& Skeen (1970). 
charge, which falls in the first hour after inoculation of the selected cells into the fresh growth medium.

The control mechanisms leading to the oscillatory oxygen uptake pattern remain to be elucidated, although the finding that cyanide (a specific inhibitor of cytochrome $c$ oxidase) does not attenuate the oscillations suggests that they are not produced by the periodic synthesis of the rate-limiting component of the respiratory chain itself. The addition of an uncoupler ( $m$-chloro-carbonylcyanide-phenyl hydrazone; $0.5 \mathrm{nmol} / \mathrm{I} \cdot 4 \times 10^{7}$ cells) gives a slight stimulation $(19 \%$ ) to an exponentially-growing culture; a similar titre of this compound does not show differential effects when added at respiratory maxima or minima (Edwards and Lloyd, unpublished results). It has been shown that the overshoot in oxygen uptake rates which gives rise to the oscillatory component of the respiration of Schizosaccharomyces pombe (Poole et al. I973) is a phenomenon peculiar to glucose-supported growth, as the peak pattern is replaced by a step pattern in glycerol-grown cells (Poole \& Lloyd, I974). Evidently the mechanisms involved in the generation of the respiratory oscillations in C. fasciculata are quite different, as glycerol serves as the major carbon and energy source in a medium which also contains a full spectrum of amino acids at high concentrations.

The timings of maxima of $\mathrm{O}_{2}$ uptake rates, adenine nucleotides, and adenylate charge in cell-cycles in several experiments are shown in Fig. 7; the periodicities of the observed oscillations were similar in cultures showing different synchrony indices. The timings of maxima of oxygen uptake rates were evidently correlated with those of adenylate charge. The consistently observed positions of maxima are summarized in a circular cell-cycle map in Fig. 8, alongside the phases of nuclear and kinetoplast DNA synthesis and organelle division. With one exception (maximum-3), close correlation between respiration and maximal pool sizes of ATP and AMP was evident. Maxima of ADP pool size occurred with a phase retardation of $36^{\circ}$ except for maximum-I (i.e. the minima observed for ADP correlated with the maxima of the two other nucleotides). All these parameters showed no obvious perturbations over the nuclear or kinetoplast S-phases. As the other two major macromolecular classes, protein and RNA, show continuous synthesis through the cell-cycle, it is clear that the balance between the supply and demand for free energy is more closely coupled to its supply than to the cellular requirements for biosynthesis. In some other synchronous systems a fall in the ATP pool accompanies the later stages of nuclear division (Chin \& Bernstein, 1968; Sachsenmaier, Scholz \& Grunet, I968), although Epel (1963) detected no changes in ATP or in ATP/ADP ratios during the S-phase of the sea urchin egg.

Adenylate charge, a more useful measure of energy balance in cells (Atkinson \& Walton, I967), has not been followed in any other system of synchronous cell growth. The concept that the viability of cells can be assessed by the measurement of this parameter (e.g. that of bacterial cells requires an adenylate charge of above 0.5; Chapman et al. 197I) would appear to be an over-simplification in the light of the present findings, as the extreme variations evident from measurements of a synchronous culture are overlooked when exponential cultures are studied (i.e. time-averaged over the duration of the cell-cycle). That the variations observed are rather slow (about $\mathrm{I} \cdot 0$ cycle/h), whereas the energyconservation reactions have time-constants of the order of seconds or less, indicates control at a different level, which is closely coupled to other events in the cell-cycle. We did not attempt to detect any high-frequency oscillations which may have been occurring as a result of control reactions with short time constants.

Synchronous cultures of trypanosomes provide an ideal system for further studies of 
the control of mitochondrial activities, the time course of mitochondrial biogenesis, and the elucidation of nucleo-mitochondrial interaction. Crithidia fasciculata has advantages over other kinetoplastidae, in that its respiration is completely cyanide-sensitive and that it has no major pathway of electron transport alternative to the phosphorylating respiratory chain (Edwards \& Lloyd, 1973).

We are indebted to Dr B. E. Brooker and Dr R. C. Brown for valuable discussions. C.E. and M.S. were holders of Science Research Council research studentships.

\section{REFERENCES}

Atrinson, D. E. \& Walton, G. M. (1967). Adenosine triphosphate conservation in metabolic regulation. Journal of Biological Chemistry 193, 265-275.

BLUMENTHAL, L. K. \& ZAHLER, S. A. (I962). Index for measurement of synchronization of cell populations. Science, New York 135, 724 .

BROOKER, B. E. (1971). Flagellar attachment and detachment of Crithidia fasciculata to the gut wall of Anopheles gambiae. Protoplasma 73, 19I-202.

Campbell, A. C. (1957). Synchronization of cell division. Bacteriological Reviews 21, 263-272.

Chapman, A. G., Fall, L. \& Atkinson, D. E. (1971). Adenylate energy charge in Escherichia coli during growth and starvation. Journal of Bacteriology 108, 1072-1086.

Chin, B. \& Bernstein, I. A. (I968). Adenosine triphosphate and synchronous mitosis in Physarum polycephalum. Journal of Bacteriology 96, 330-337.

Chiu, S. V., Kao, I. C., Erickson, L. E. \& Fan, L. T. (1973). ATP pools in activated sludge. Journal of Water Pollution Control Federation 45, 1746-1758.

Cosgrove, B. \& Skeen, M. J. (1970). The cell-cycle in Crithidia fasciculata. Temporal relationships between synthesis of deoxyribonucleic acid in nucleus and in the kinetoplast. Journal of Protozoology 17, 172177 .

Edwards, C. \& Lloyd, D. (1973). Terminal oxidases and carbon monoxide-reacting haemoproteins in the trypanosomatid, Crithidia fasciculata. Journal of General Microbiology 79, 275-284.

EPEL, D. (1963). The effects of carbon monoxide inhibition on ATP level and the rate of mitosis in the sea urchin egg. Journal of Cell Biology 17, 315-319.

Herbert, D., Phipps, P. J. \& Strange, R. E. (1971). Chemical analysis of microbial cells. In Methods in Microbiology, vol. 5B, pp. 209-344. Edited by J. R. Norris and D. W. Ribbons. London and New York: Academic Press.

LLoYD, D. (1974). Mitochondria of Microorganisms. London, New York: Academic Press.

Lloyd, D. \& Brookman, J.S. G. (1967). An oxygen electrode reaction vessel. Biotechnology and Bioengineering 9, 271-272.

Lloyd, D., John, L., Edwards, C. \& Chagla, A. H. (1975). Synchronous cultures of micro-organisms: large-scale preparation by continuous-flow size selection. Journal of General Microbiology 88, 153-158.

Mitchison, J. M. (1971). The Biology of the Cell Cycle. Cambridge University Press.

Mitchison, J. M. \& Vincent, W. S. (1965). Preparation of synchronous cell cultures by sedimentation. Nature, London 205, 987-989.

Newton, B. A. (1957). The mode of action of phenanthridines: the effect of ethidium bromide on cell division and nucleic acid synthesis. Journal of General Microbiology 17, 71 8-730.

Poole, R. K. \& LLOYD, D. (I974). Changes in respiratory activities during the cell-cycle of the fission yeast, Schizosaccharomyces pombe $97 \mathrm{~h}^{-}$, growing in the presence of glycerol. Biochemical Journal $\mathbf{4 4 4}$, I4I-148.

Poole, R. K., LloYD, D. \& KemP, R. B. (1973). Respiratory oscillations and heat evolution in synchronously dividing cultures of the fission yeast, Schizosaccharomyces pombe $972 \mathrm{~h}^{-}$. Journal of General Microbiology 77, 209-220.

Sachsenmaier, W., Scholz, R. \& Grunet, J. (1968). Änderungen des Nucleotid-Spiegels im Verlauf des synchronen Mitosezyklus von Physarum polycephalum. Hoppe-Seyler's Zeitschrift für physiologische Chemie 349, 1257.

Simpson, L. (1972). The kinetoplast of the Hemoflagellates. International Review of Cytology 32, 139-207. 
Simpson, L. \& Braly, P. (1970). Synchronization of Leishmania tarentolae by hydroxyurea. Journal of Protozoology 17, 5I I-5I 7 .

Steinert, M. (1969). Reversible inhibition of the division of Crithidia luciliae by hydroxyurea and its use for obtaining synchronized cultures. FEBS Letters 5, 29I-294.

Steinert, M. \& Steinert, G. (1962). La synthèse de l'acide désoxyribonucléique au cours du cycle de division de Trypanosoma mega. Journal of Protozoology 9, 203-2I I.

SteinerT, M. \& VAN Assel, S. (1967). Replications coordonées des acides désoxyribonucléiques nucleaire et mitochondrial chez Crithidia luciliae. Archives internationales de physiologie et de biochimie 75, 370-37I.

StuART, K. \& Hanson, E. D. (1967). Acriflavin induction of dyskinetoplasy in Leptomonas karyophilus. Journal of Protozoology 14, 39-43.

Van Assel, S. \& Steinert, G. (1971). Nuclear and kinetoplastic DNA replication cycles in normal and synchronously dividing Crithidia luciliae. Experimental Cell Research 65, 353-358. 